\title{
The presence of Interleukin-13 in nasal lavage may be a predictor of nasal polyposis in pediatric patients with cystic
} fibrosis*

\author{
Jamil Manji ${ }^{1,2}$, Andrew Thamboo', Mark Tacey ${ }^{2,3}$, Cathie Garnis ${ }^{4}$, \\ Neil K. Chadha' \\ ' Division of Pediatric Otolaryngology-Head and Neck Surgery, B.C. Children's Hospital, Vancouver, British Columbia, Canada \\ 2 Department of Medicine, University of Melbourne, Melbourne, Victoria, Australia \\ ${ }^{3}$ Northern Centre for Health Education and Research, Northern Hospital, Melbourne, Victoria, Australia \\ ${ }^{4}$ Division of Otolaryngology, Department of Surgery, University of British Columbia, Vancouver, British Columbia, Canada
}

Rhinology 56: 3, 261-267, 2018

https://doi.org/10.4193/Rhin17.007

*Received for publication:

January 10, 2017

Accepted: February 8, 2018

Background: Sinonasal disease is a common feature of cystic fibrosis (CF) and can cause significant morbidity in these patients. Our objective was to determine if CF individuals with concomitant nasal polyposis (NP) express a unique profile of inflammation and if so, whether these inflammatory cytokine mediators have predictive value in identifying these individuals for prompt management by an Otolaryngologist.

Methodology: Nasal lavage samples and clinical outcomes of disease severity were obtained from thirty-eight pediatric CF individuals. Participants were subdivided based on the presence or absence of NP. Nasal lavage samples were analyzed on a panel of seventeen cytokine targets using a Bio-Plex Luminex assay. A Perl Permutation test with correction for multiple hypotheses was performed to identify uniquely expressed cytokines between CF individuals with NP (CFwNP) and those without (CFsNP).

Results: Thirty-five patients were included in the analysis. Cytokines IL-13 and GM-CSF were uniquely expressed in the CFwNP group when compared to the CFsNP group. Logistic regression analysis demonstrated a significant association of IL-13 with NP.

Conclusion: In children diagnosed with CF, the level of IL-13 in nasal lavage samples could potentially serve as a non-invasive clinical tool in predicting NP in this population, and a target for future immunotherapy.

Key words: cystic fibrosis, nasal polyps, sinusitis, immunology, cytokines

\section{Introduction}

Great strides have been made in the treatment of cystic fibrosis (CF) over the last several decades, affording CF individuals profound improvements in quality of life and life expectancy ${ }^{(1)}$. Unfortunately, advancement in the management of lower respiratory tract disease and the systemic manifestations of $\mathrm{CF}$ may be compromised by the unchecked progression of concomitant sinonasal disease in these individuals. More than $90 \%$ of CF individuals demonstrate radiological evidence of sinus disease ${ }^{(2)}$ and approximately $50 \%$ also suffer from concomitant nasal polyposis ${ }^{(3)}$. Nasal polyposis may develop as early as the preschool period ${ }^{(4)}$, unfortunately, CF individuals with nasal polyposis (CFwNP) may not seek out specialist care until their sinonasal symptoms reach severe proportions. Nasal polyposis is traditionally classified as either eosinophilic TH2-mediated inflammation or neutrophilic TH1-mediated inflammation, the latter form associated with $\mathrm{CF}^{(3)}$. The cystic fibrosis transmembrane receptor (CFTR) gene mutation associated with CF results in defective ion transportation in mucosal glands of both the upper and lower respiratory tract. The production of more viscous secretions impairs sinonasal mucociliary clearance, creating an environment conducive to chronic infection and severe neutrophil-dominated chronic inflammation ${ }^{(4)}$. However, the specific pathogenesis of nasal polyposis in CF and why it occurs in only a subset of patients is poorly understood. Previous investigations have identified cytokines IL-6, IL-8, IL-10, and IL-1 $\beta$ as being present in the nasal lavage of CF individuals ${ }^{(5)}$. Identification and quantification of these cytokines were found to be 
sensitive in discriminating between periods of exacerbation and clinical stability in CF (5).

CF patients are commonly managed by Respirologists and only referred to an Otolaryngologist if there is evidence of frank nasal polyposis or if the patient has complained of symptoms consistent with sinonasal disease. Despite the high prevalence of CFwNP, it may not be practicable or feasible for Otolaryngologists to see all CF patients in a timely manner. Therefore, affected individuals and their healthcare teams may benefit from predictive markers of nasal polyposis to assist in more urgent referral of CF patients with a higher likelihood of having severe sinonasal disease. Thamboo et al illustrated that a score greater than 11 on the Sinonasal Outcome Test-22 (SNOT-22) is predictive of nasal polyposis in children with $\mathrm{CF}^{(6)}$. Despite the facility of the SNOT-22, a medical assistant or engaged parent is often needed to assist pediatric patients with answering questions. Nasal saline lavages are frequently performed for therapeutic purposes in children suffering from CF. Rinsing the nasal and sinus cavities with saline solution can assist mucus clearance, potentially reducing symptoms associated with the increased mucus viscosity and the chronic inflammation. We sought to determine whether inflammatory mediator proteins present in nasal lavage samples of pediatric CF individuals could be predictive of the presence of nasal polyposis. We hypothesized that the cytokine profile of nasal lavage fluid may be predictive of the presence or absence of nasal polyposis, and if a consistent profile could be identified, it may have utility in developing a "litmus test" to help identify CFwNP in a clinical setting. Additionally, future immunotherapeutic options may be possible by targeting specific inflammatory cytokines or pathways that are altered in CFwNP.

\section{Materials and methods}

This cross-sectional study was conducted under the auspices of the University of British Columbia Children's and Women's Research Ethics Board (H10-03132). The following criteria were used to enroll and exclude patients from the study: patients with a confirmed diagnosis of CF based on genetic testing; between the ages of 6 and 18; not actively being managed by the Pediatric Otolaryngology Department; and willing to provide a nasal lavage sample for cytokine analysis. All baseline characteristics and several clinical data points were obtained in the same patient population from our previous study ${ }^{(6)}$. The presence or absence of NP was determined by an Otolaryngologist with a 2.4-mm flexible endoscope. The associated SNOT-22 scores and nasal bacterial colonization data from our original study of this population was also included ${ }^{(6)}$.

\section{Nasal lavage}

Nasal lavage has been validated as a viable method of collecting biological samples suitable for cytokine analysis in lieu of col- lecting sinus tissue ${ }^{(7)}$. A 12-French Foley catheter was gently inserted into one nasal cavity. If patients had unilateral NP, the distal end of the catheter was placed on the side of the polyps. The catheter balloon was then inflated so that the catheter sat comfortably within the internal nasal valve and the balloon formed a seal external to the nostril. Participants were instructed to place their chin to their chest, with their head in a flexed position. With the contralateral nostril pinched and holding their breath, $10 \mathrm{cc}$ of sterile normal saline was pumped via a syringe through the catheter back and forth three times. The lavage was then collected through the catheter with the syringe. The samples were immediately placed on ice and transferred to the laboratory to be centrifuged. The supernatant was collected and stored at $-70^{\circ} \mathrm{C}$ to be analyzed as a batch of samples. Samples were analyzed for cytokine levels as per manufacturers protocol using the Human Cytokine 17-Plex Antibody Bead Kit (Bio-Rad Laboratories, CA, USA).

\section{Cytokine analysis}

The prevalence of 17 cytokines and total protein were quantified for each sample with a Luminex and cytokine multiplex assay (Bio-Rad Laboratories) platform. Nasal lavage samples were assayed in duplicate as described in similar studies ${ }^{\left({ }^{8}\right)}$. The cytokine assay included a panel of probes specific to: Human IL-1 $\beta$, IL-2, IL-4, IL-5, IL-6, IL-7, IL-8, IL-10, IL-12, IL-13, IL-17, G-CSF, GM-CSF, IFN- $\gamma$, MCP-1, MIP-1 $\beta$, and TNF- $\alpha$. Total protein was calculated for each sample using a standard BCA Assay (Fischer Scientific Inc.). For comparison across groups and across patients, cytokine levels were normalized to total protein as described in similar studies ${ }^{(9)}$. Cytokine expression was reviewed to determine if it was consistent with findings reported in the literature.

\section{Statistical analysis}

The predictive value for the presence of NP was calculated using the cytokine data obtained from Luminex analysis of nasal lavage samples collected in clinic. Cytokine biomarker candidates were identified using the Perl Permutation Test (10), a non-parametric test of the means between two different populations. The Perl Permutation Test is optimally suited to the task of identifying unique genes and protein products because it does not make assumptions about the normality of the underlying distribution. The number of samples required for the test to achieve adequate statistical power is relatively low (compared to t-test and chi-squared test) and each additional sample increases the power of the test exponentially. For each cytokine a permutation score is produced which denotes how likely the actual observed difference between study groups occurs by chance. In the context of this study, the larger the permutation score the more significant the observed difference between CFwNP and CF without nasal polyposis (CFsNP) individuals. The converse is that cytokines with a permutation score near zero would serve 
Table 1. Non-parametric permutation analysis to distinguish cytokines expressed uniquely between CFwNP and CFsNP cohorts.

\begin{tabular}{|cccc|}
\hline Cytokine & $\begin{array}{c}\text { Permutation } \\
\text { Score }\end{array}$ & SD & p-value \\
\hline IL-13 & 6.15 & $6.77 \mathrm{E}-05$ & $<0.001$ \\
\hline GM-CSF & 5.52 & $5.90 \mathrm{E}-04$ & $<0.001$ \\
\hline IL-4 & 0.07 & $5.77 \mathrm{E}-05$ & 0.47 \\
\hline IL-5 & 0.49 & $2.29 \mathrm{E}-05$ & 0.41 \\
\hline IL-6 & 0.33 & $7.75 \mathrm{E}-4$ & 0.42 \\
\hline IL-8 & 0.88 & $1.71 \mathrm{E}-2$ & 0.29 \\
\hline MCP-1 & 0.39 & $6.32 \mathrm{E}-4$ & 0.42 \\
\hline TNF- $\alpha$ & 0.79 & $9.43 \mathrm{E}-05$ & 0.30 \\
\hline
\end{tabular}

Abbreviations: CFwNP=Cystic Fibrosis with Nasal Polyposis;

CFsNP=Cystic Fibrosis without Nasal Polyposis

as ideal reference cytokines for follow-up validation studies.

Correcting for multiple hypotheses, a $\mathrm{p}$-value of $\mathrm{x}<0.001$ was considered significant. Cytokines identified by the permutation test were further evaluated with a non-parametric, unpaired t-test (Mann-Whitney test) of the means in each disease group (GraphPad Prism v6.0, GraphPad Software, CA, USA). An alpha value of 0.05 was used to determine significance.

Cytokine prevalence was dichotomized by establishing a receiver-operating characteristic $(\mathrm{ROC})$ curve for the presence or absence of nasal polyposis (STATA version 12.1, StataCorp, College Station, TX, USA). Univariate logistic regression analysis was used to determine the association between nasal polyposis and each clinical variable, with variables considered for multivariate analysis if the $p$-value $<0.20$. Using a forward step-wise regression technique, potential predictors were included in the logistic regression model. An alpha value of 0.05 was used to determine significance. Results were reported as Odds Ratios (OR) with associated $95 \%$ Confidence intervals.

\section{Results}

Thirty-eight patients consented to provide nasal lavage samples, of which thirty-five samples were viable $(n=35)$. Non-viable samples were those resulting in little or no protein content upon analysis. The average age of recruited patients was 11.7 years old (SD 3.2). The incidence of NP in this cohort of pediatric CF subjects was $51 \%(n=18)$, which is consistent with the range reported in the literature. Six of these subjects had overt (Grade III) nasal polyps ${ }^{(11)}$. Among CFwNP individuals, a minority reported sinus symptoms upon query: facial pain $(n=1)$, nasal obstruction $(n=8)$, and reduced sense of smell $(n=3)$. Six CFwNP subjects reported one of these symptoms in isolation. Ten CFwNP subjects reported no symptoms of facial pain, nasal obstruction, or hyposmia. Nasal swab culture demonstrated bacterial colonization
Table 2. Univariate association of clinical and cytokine variables with nasal polyposis (NP).

\begin{tabular}{|c|c|c|c|}
\hline \multirow[t]{2}{*}{ Variable } & \multicolumn{2}{|c|}{ NP } & \multirow[b]{2}{*}{ p-value } \\
\hline & No & Yes & \\
\hline Patients & 17.0 & 18.0 & \\
\hline Female & $7.0(41)$ & $6.0(33)$ & 0.631 \\
\hline Age, Mean $\pm S D$ & $11.6 \pm 3.3$ & $12.0 \pm 3.1$ & 0.749 \\
\hline $\begin{array}{l}\text { SNOT-22 Score, Med } \\
\text { (IQR) }\end{array}$ & $7.0(2-11)$ & $14.0(11-24)$ & 0.002 \\
\hline SNOT-22 Score > 11 & $4.0(23)$ & $13.0(72)$ & 0.007 \\
\hline IL-13, Med (IQR) & $\begin{array}{c}2.3 \mathrm{E}-4 \\
(0-3.8 \mathrm{E}-4)\end{array}$ & $\begin{array}{c}3.0 E-4 \\
(1.5 E-4-4.3 E-4)\end{array}$ & 0.144 \\
\hline $\mathrm{IL}-13>8.79 \mathrm{E}-5$ & $9.0(53)$ & $16.0(90)$ & 0.027 \\
\hline GM-CSF, Med (IQR) & $\begin{array}{c}5.1 E-3 \\
(4.2 E-3-6.6 E-3)\end{array}$ & $\begin{array}{c}5.5 E-3 \\
(4.7 E-3-6.9 E-3)\end{array}$ & 0.575 \\
\hline GM-CSF > 5.14E-3 & $7.0(41)$ & $12.0(67)$ & 0.130 \\
\hline
\end{tabular}

n (\%) unless otherwise specified

in eight CFwNP subjects (all with Staphyloccus aureus) and eight CFsNP subjects (7 with Staphylococcus aureus, 1 with Pseudomonas aeruginosa).

Expression of IL-13 and GM-CSF were distinguishing molecular features between CFwNP and CFsNP (Table 1). IL-13 was expressed significantly higher in patients with nasal polyposis ( $p<0.001$, permutation score: 6.15). The cytokine GM-CSF was expressed significantly higher in patients without nasal polyposis ( $p<0.001$, permutation score: 5.52 ). Non-parametric unpaired Mann-Whitney test of IL-13 expression between CFwNP and CFsNP cohorts was not significant $(p=0.23)$. However, the mean normalized expression of IL-13 in CFwNP cases (2.92E-4 \pm

2.14E-4) was approximately $40 \%$ higher than those with CFsNP (2.09E-4 $\pm 1.97 \mathrm{E}-4)$.

In accordance with our previous work showing correlation of SNOT-22 score with the presence of nasal polyposis ${ }^{(6)}$, IL-13 was similarly up-regulated in patients with a severe SNOT-22 score. The non-parametric PERL permutation test demonstrated that IL-13 was significantly higher in individuals with SNOT-22 score greater than 11 (permutation score $=5.97 ; p<0.001$ ).

The cytokines IL-4, MIP-1 $\beta$, IL- 6 and IL- 8 were expressed in similar proportions across CFwNP and CFsNP subjects (Table 1). Among these, IL-4 was found to have a permutation score near zero (Table 1), indicating that there was a negligible difference in expression of IL-4 between CFwNP and CFsNP groups. This quality identified IL-4 as an ideal reference cytokine for comparing IL-13 and GM-CSF expression among a mixed cohort of CFwNP and CFsNP individuals.

ROC analysis (Figure $1 \mathrm{~A}$ and 1B) identified a cut-off of $8.79 \mathrm{E}-5$ in 

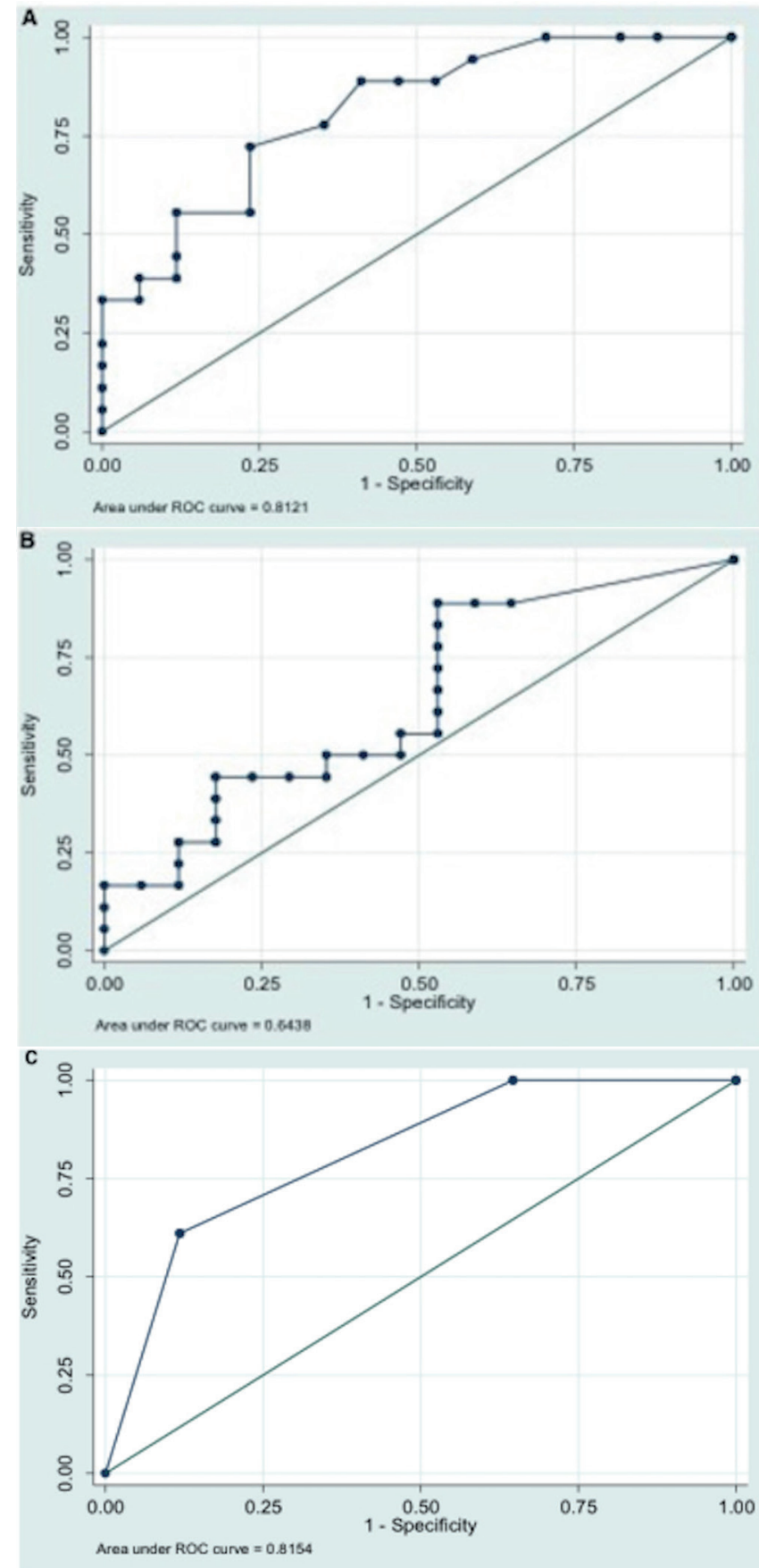

Figure 1. Receiver operating characteristic (ROC) curve of A) SNOT-22 score B) IL-13 level and C) final predictive model, based on IL-13 level and SNOT-22 score, to estimate the probability of suffering from CF with concomitant nasal polyposis.

the IL-13 values that provided an independently significant association with CFwNP (Sensitivity $88.89 \%$, Specificity $47.06 \%$, OR 9.94, 95\% Cl: 1.23-80.37) along with the SNOT-22 score (Sensitivity $77.78 \%$, Specificity $64.71 \%$, OR $10.97,95 \%$ Cl: $1.84-65.45)$, with a cut-off of 11 also being determined from ROC analysis (Table 3b). GM-CSF based on a cut off of 5.14E-3 was not independently associated with NP in addition to the SNOT-22
Table 3. Predicted probability of cytokine and clinical variables for determining nasal polyposis risk.

Table 3a - Univariate associations with NP, expressed as odds ratio

\begin{tabular}{|lccc|}
\hline Variable & \multicolumn{3}{c}{ NP } \\
\hline SNOT-22 $>11$ & OR & $\mathbf{9 5 \% ~ C l}$ & p-value \\
\hline
\end{tabular}

Table 3b - Multivariate associations with NP, including cytokine variables (binary)

\begin{tabular}{|c|c|c|c|}
\hline \multirow{2}{*}{ Variable } & \multicolumn{3}{|c|}{ NP } \\
\hline & OR & $95 \% \mathrm{Cl}$ & p-value \\
\hline SNOT-22 > 11 & 10.97 & $1.84-65.45$ & 0.009 \\
\hline IL-13 > 8.79E-5 & 9.94 & $1.23-80.37$ & 0.031 \\
\hline \multicolumn{4}{|c|}{$\begin{array}{l}\text { Table 3c - Multivariate associations with NP, including cytokine } \\
\text { variables (binary) }\end{array}$} \\
\hline \multirow{2}{*}{ Variable } & \multicolumn{3}{|c|}{ NP } \\
\hline & OR & $95 \% \mathrm{Cl}$ & p-value \\
\hline SNOT-22 > 11 & 8.95 & $1.81-44.30$ & 0.007 \\
\hline GM-CSF $>5.14 \mathrm{E}-3$ & 3.17 & $0.64-15.68$ & 0.158 \\
\hline \multicolumn{4}{|c|}{$\begin{array}{l}\text { Table } 3 \mathrm{~d} \text { - Multivariate associations with NP, including cytokine } \\
\text { variables plus age/gender }\end{array}$} \\
\hline \multirow{2}{*}{ Variable } & \multicolumn{3}{|c|}{ NP } \\
\hline & OR & $95 \% \mathrm{Cl}$ & p-value \\
\hline SNOT-22 > 11 & 12.00 & $1.91-75.35$ & 0.008 \\
\hline $\mathrm{IL}-13>8.79 \mathrm{E}-5$ & 10.14 & $1.16-89.03$ & 0.037 \\
\hline Female & 0.89 & $0.15-5.41$ & 0.896 \\
\hline Age & 1.10 & $0.83-1.46$ & 0.490 \\
\hline
\end{tabular}

score (Table 3c), with gender and age also not independently associated with NP, when included in a model with IL-13 and SNOT-22 scores (Table 3d). Based on the final multivariate model (Figure 1C), a 3-level scoring system was developed to indicate whether a patient had SNOT-22 less than 11 and IL-13 less than 8.79E-5 (Score: 0); either SNOT-22 more than 11 or IL-13 more than $8.79 \mathrm{E}-5$ (Score: 1 ) or both SNOT-22 more than 11 and IL-13 more than $8.79 \mathrm{E}-5$ (Score: 2 ). The ROC analysis indicates that the predictive performance is not significantly improved through the addition of IL-13 to the SNOT-22 score for the prediction of NP (C-stat improved from 0.812 to $0.815, \mathrm{p}=0.96$ ).

Assessing the relationship between cytokine expression and clinical variables in this cohort revealed an association between IL-8 expression and acute bacterial infection of the nose. Mean normalized expression of IL-8 in individuals with nasal culture positive for $S$. aureus or $P$. aeuriginosa $(\mu=1.33 \mathrm{E}-1 \pm 1.15 \mathrm{E}-1)$ was $73 \%$ higher than in those who tested negative for these bacteria $(9.55 \mathrm{E}-2 \pm 9.91 \mathrm{E}-2)$. Analysis of the distribution with the non- 
parametric unpaired Mann Whitney test demonstrated no significant difference between the two cohorts $(p=0.14)$. The mean normalized expression of IL-8 was found to be similar between CFsNP (1.04E-1 $\pm 8.08 \mathrm{E}-2$ ) and CFwNP individuals (9.55E-2 $\pm 9.91 \mathrm{E}-2)$. This difference was also found to be non-significant $(p=0.36)$. There were no other notable associations found between cytokines and clinical variables in this population.

\section{Discussion}

Our findings demonstrated marked differences in the inflammatory profile between CFwNP and CFsNP. Two cytokines in particular, IL-13 and GM-CSF, were found to be significantly different between the groups based on analysis with a nonparametric Perl Permutation test. These cytokines represented the most unique and differentiating inflammatory features of each disease category.

In CF animal models, IL-13 is highlighted as a cytokine of interest for its role in perpetuating the chronic inflammatory processes in the gut and lower respiratory tract ${ }^{(12,13)}$. The constitutive expression of IL-13 in murine intestinal epithelial cells induced villous atrophy, goblet cell hyperplasia and mucous hypersecretion ${ }^{(13)}$. Most importantly, these changes in epithelial architecture were accompanied by increased secretion of chlorine ions (Cl-) and were associated with an increase in CFTR expression. Similarly, in the lower respiratory tract, IL-13 is a driver of goblet cell hyperplasia, but also contributes to airway hyper-responsiveness via the modulation of smooth muscle calcium channels (independent of eosinophils and $\lg E)^{(12)}$.

Despite the reduced presence of eosinophils in CFwNP compared with allergic rhinitis with nasal polyposis, it is known that eosinophils still play a critical role in driving the development of nasal polyposis ${ }^{(14)}$. In a study of nasal polyp tissue collected from CFwNP individuals, an eosinophilic infiltrate comprised $45 \%$ of samples, of which $80 \%$ also contained a mixed neutrophilic infiltrate ${ }^{(15)}$. The release of IL-13 by human eosinophils in eosinophilic inflammatory responses is also well characterized ${ }^{(16)}$. In vitro findings suggest that IL-13 production is a cytokine-driven process, induced by IL-5 or GM-CSF stimulation of eosinophils. In vivo analysis of nasal tissue found that IL-13 is also released in response to common nasal allergen provocation, along with a marked increase in tissue eosinophilia, and this response was sustained up to one week from the provocation event ${ }^{(17)}$. Consistent with this mechanism, IL-13 was significantly upregulated in this population of CFwNP compared to CFsNP individuals. Upon analysis of these groups using non-parametric unpaired Mann Whitney test, the association of IL-13 with nasal polyposis status was found to be not significant in the current study. It is frequently the case in biomedical research where small sample sizes and the inclusion of multiple analytes make for insufficient statistical power to test for associations using traditional non-parametric tests. The permutation test is better suited for the identification of gene and protein products because it does not make assumptions about the normality of the underlying distribution ${ }^{(10)}$.

As Table 1 indicates, several inflammatory cytokines were also similarly expressed in both CFwNP and CFsNP. Among those cytokines expressed similarly between study cohorts in this investigation, IL- 8 is was notable. Previous studies have associated IL-8 with the neutrophilic inflammation characteristic of CFwNP

(2). Interestingly, recent literature has disputed this association. Nunes et al. found that elevated levels of IL-8 seen in CFwNP were more likely associated with the presence of an acute bacterial infection (P. aeruginosa and/or S. aureus) in CF patients, rather than polyp status ${ }^{(18)}$. In CFwNP groups where nasal swab samples were culture negative for S. aureus, IL-8 was expressed at much lower levels in their study ${ }^{(18)}$. Our results corroborated these findings. Individuals with positive nasal culture for Staphylococcus aureus or Pseudomonas aeruginosa at the time of examination had higher levels of IL-8 on average than those that were culture negative. As the proportion of those with active infection was well balanced between CFwNP and CFsNP cohorts, IL-8 was not found to be significantly different between these groups (permutation score $=0.88 ; p=0.29$ ) (Table 1). A predictive model with higher sensitivity at the expense of specificity was favored since the gold standard for confirmation of nasal polyposis, flexible endoscopy of the nasal cavity, can be performed quite easily by an Otolaryngologist, and causes little distress to the patient. The prospect of nasal polyposis left untreated in a CF individual poses a much greater risk. The impact of nasal polyposis on quality of life can be extremely detrimental and may produce symptoms such as nasal obstruction, mouth breathing, headache, rhinorrhea, facial pain, anosmia, halitosis, and restless sleep ${ }^{(3)}$. If left untreated, the expansion of nasal polyps may also cause facial deformities and visual disturbance (3). Obstruction of the nasal cavity by polypoid oedema presents a challenge to treatment by restricting access to the mucosa for nasal-administered corticosteroid rescue medication, contributing to the medically refractory nature of CFwNP. These patients tend not to respond to conventional corticosteroid treatment as effectively as those with eosinophilic nasal polyposis ${ }^{(3)}$. When medical management options have been exhausted, surgical removal of nasal polyps may be indicated.

Thamboo et al. demonstrated that a subjective clinical evaluation tool, the sinonasal outcomes test-22 (SNOT-22) could effectively identify CF pediatric patients at high risk of suffering from concomitant nasal polyposis ${ }^{(6)}$. Setting a threshold SNOT22 score of 11 for detecting CFwNP in their study, the positive predictive value was $68.1 \%$, the negative predictive value was $66.7 \%$, and the positive likelihood ratio was 1.82 . In accordance with the clinical trial conducted by Thamboo et al., elevated levels of IL-13 in our tissue samples were correlated with SNOT-22 score greater than 11 (Table 2). While the ROC analysis 
indicated that the predictive performance was not significantly improved through the addition of IL-13 to the SNOT-22 score for the prediction of NP, this may be due to the limited sample size in this study preventing an exploration of multiple thresholds for each variable (IL-13 and SNOT-22) on their continuous scale. The limited sample size also did not allow evaluation or validation of a scoring system that could be clinically valuable in predicting the likelihood of NP for this patient group. At present, administering the SNOT-22 questionnaire is a more feasible and sensitive method of identifying CF candidates for tertiary care of NP. However, further identification of these unique features of the sinonasal inflammatory profile could go beyond early identification of CFwNP individuals and form the basis for targeted, personalized immunotherapy.

Unfortunately, while comparable in size to other similar studies, this investigation was limited by its small sample size. This may have limited the ability to observe stronger associations; conversely, it may have distorted our findings. Future investigations should aim to collect serial nasal lavage samples from CF participants in order to appreciate the dynamics of the inflammatory profile during periods of clinical stability and during exacerbations of sinonasal disease. Because this study only captured a cross-sectional snapshot of the inflammatory profile at one clinic visit, it is possible that other potential candidate cytokine biomarkers were overlooked. Given that $\mathrm{CT}$ evidence of sinus disease was not available for these subjects, it is also possible that cytokine profile representing the control group of CFsNP may be reflective of a mixed cohort of CF children with CRS and a much smaller proportion without CRS. Follow-up investigations aiming to validate the results of this study should focus on specific targets for IL-13 and GM-CSF, as well as a reference cytokine such as IL-4. Efforts should be made to also include upstream analysis of mRNA and protein expression of Toll-like receptor's (TLR) associated with these cytokines. Aberrant expression of TLR's has previously been associated with allergic $N P^{(19)}$ and may shed more light on the specific mechanism of pathogenesis in CFwNP.

\section{Conclusion}

The expression of inflammatory cytokines in nasal lavage samples could serve as a minimally invasive tool to identify paediatric cystic fibrosis individuals at higher risk of developing concomitant nasal polyposis. Specifically, the presence of IL-13 in nasal secretions shows strong positive correlation with nasal polyposis in cystic fibrosis. Further work into elucidating the inflammatory profile associated with cystic fibrosis with nasal polyposis could aid in early treatment and potentially, the development of novel, personalized medicine for this disease population.

\section{Acknowledgements}

We gratefully acknowledge the contributions of our Research Coordinators, Rachelle Dar Santos and Julie Pauwels, in seeing this project to completion; and BC Children's Hospital pediatric cystic fibrosis clinic for accommodating our study during clinic hours. None of these individuals received compensation for their contributions. External funding was provided by the Rare Diseases Foundation and the Child and Family Research Institute Innovations in Acute Care \& Technology (iACT) grant.

\section{Authorship contribution}

JM: Study concept and design; Obtaining funding; Acquisition, analysis, and interpretation of data; Drafting of the manuscript; Critical revision of the manuscript for important intellectual content; AT: Study concept and design; Obtaining funding; Interpretation of data; Drafting of the manuscript; Critical revision of the manuscript for important intellectual content; MT: Analysis of data; Drafting of the manuscript; CG: Study concept and design; Obtaining funding; Critical revision of the manuscript for important intellectual content; Study supervision; NKC: Study concept and design; Obtaining funding; Critical revision of the manuscript for important intellectual content; Study supervision.

\section{Conflict of interest}

The authors have no conflicts of interest to declare. The Rare Diseases Foundation and Child and Family Research Institute had no role in the design and conduct of the study; collection, management, analysis, and interpretation of the data; preparation, review, or approval of the manuscript; and decision to submit the manuscript for publication.

\section{References}

1. Clancy JP, Jain M. Personalized medicine in cystic fibrosis: dawning of a new era. Am J Respir Crit Care Med. 2012; 186:593-597.

2. Sobol SE, Christodoulopoulos P, Manoukian JJet al. Cytokine profile of chronic sinusitis in patients with cystic fibrosis. Arch Otolaryngol Head Neck Surg. 2002; 128:1295-1298

3. Chaaban MR, Walsh EM, Woodworth BA
Epidemiology and differential diagnosis of nasal polyps. Am J Rhinol Allergy. 2013 27:473-478.

4. Mainz JG, Koitschev A. Pathogenesis and management of nasal polyposis in cystic fibrosis. Curr Allergy Asthma Rep. 2012; 12:163-174.

5. Paats MS, Bergen IM, Bakker Met al. Cytokines in nasal lavages and plasma and their correlation with clinical parameters in cystic fibrosis. J Cyst Fibros. 2013; 12:623629

6. Thamboo A, Santos RC, Naidoo L, Rahmanian R, Chilvers MA, Chadha NK. Use of the SNOT-22 and UPSIT to appropriately select pediatric patients with cystic fibrosis who should be referred to an otolaryngologist: cross-sectional study. JAMA Otolaryngol Head Neck Surg. 2014; 140:934939 
7. Oyer SL, Mulligan JK, Psaltis AJ, Henriquez OA, Schlosser RJ. Cytokine correlation between sinus tissue and nasal secretions among chronic rhinosinusitis and controls. Laryngoscope 2013; 123:E72-78.

8. Bennett BL, Garofalo RP, Cron SGet al. Immunopathogenesis of respiratory syncytial virus bronchiolitis. J Infect Dis. 2007 195:1532-1540.

9. Repka-Ramirez S, Naranch K, Park YJ, Clauw D, Baraniuk JN. Cytokines in nasal lavage fluids from acute sinusitis, allergic rhinitis, and chronic fatigue syndrome subjects. Allergy Asthma Proc. 2002; 23:185-190.

10. Chari R, Lonergan KM, Pikor LAet al. A sequence-based approach to identify reference genes for gene expression analysis. BMC Med Genomics 2010; 3:32.

11. Lund VJ, Kennedy DW. Staging for rhinosinusitis. Otolaryngol Head Neck Surg 1997; 117:S35-40.

12. McCuaig S, Martin JG. How the airway smooth muscle in cystic fibrosis reacts in proinflammatory conditions: implications for airway hyper-responsiveness and asthma in cystic fibrosis. Lancet Respir Med 2013; 1:137-147.

13. Wu D, Ahrens R, Osterfeld Het al.
Interleukin-13 (IL-13)/IL-13 receptor alpha1 (IL-13Ralpha1) signaling regulates intestinal epithelial cystic fibrosis transmembrane conductance regulator channel-dependent Cl- secretion. J Biol Chem 2011; 286:1335713369.

14. Jankowski R, Bouchoua F, Coffinet L, Vignaud JM. Clinical factors influencing the eosinophil infiltration of nasal polyps. Rhinology 2002; 40:173-178.

15. Steinke JW, Payne SC, Chen PG, Negri J, Stelow EB, Borish L. Etiology of nasal polyps in cystic fibrosis: not a unimodal disease. Ann Otol Rhinol Laryngol. 2012; 121:579586.

16. Schmid-Grendelmeier P, Altznauer F, Fischer Bet al. Eosinophils express functional IL-13 in eosinophilic inflammatory diseases. J Immunol 2002; 169:1021-1027.

17. KleinJan A, Dijkstra MD, Boks SS, Severijnen LA, Mulder PG, Fokkens WJ. Increase in IL-8, IL-10, IL-13, and RANTES mRNA levels (in situ hybridization) in the nasal mucosa after nasal allergen provocation. J Allergy Clin Immunol 1999; 103:441-450.

18. Nunes FB, Castro MC, Silva TM et al. Cytokine profile in subjects with Cystic Fibrosis and nasal polyposis compared to patients with no nasal disorders. Braz J Otorhinolaryngol. 2010; 76:25-28.

19. Ramanathan M, Jr., Lee WK, Dubin MG, Lin S, Spannhake EW, Lane AP. Sinonasal epithelial cell expression of toll-like receptor 9 is decreased in chronic rhinosinusitis with polyps. Am J Rhinol 2007; 21:110-116.

Dr. Neil K. Chadha

Division of Pediatric Otolaryngology-

-Head and Neck Surgery

B.C. Children's Hospital

K2-184, 4480 Oak Street

Vancouver

British Columbia

Canada, V6H 3V4

Tel: +1-604-875-3730

E-mail: nchadha@cw.bc.ca 